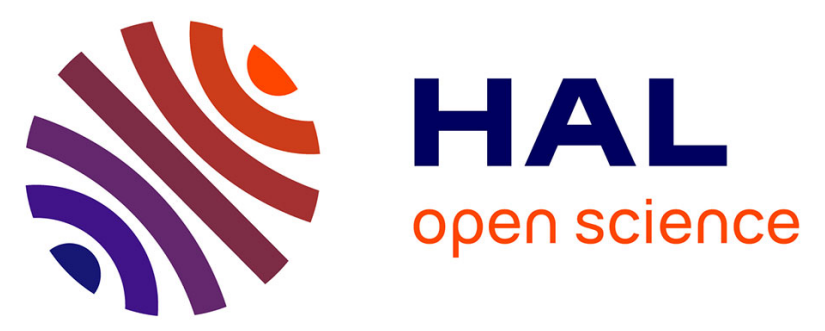

\title{
Using formal concept analysis for checking the structure of an ontology in LOD: the example of DBpedia
}

Pierre Monnin, Mario Lezoche, Amedeo Napoli, Adrien Coulet

\section{To cite this version:}

Pierre Monnin, Mario Lezoche, Amedeo Napoli, Adrien Coulet. Using formal concept analysis for checking the structure of an ontology in LOD: the example of DBpedia. 23rd International Symposium on Methodologies for Intelligent Systems, ISMIS 2017, Warsaw university of technology, Jun 2017, Warsaw, Poland. pp.674-683, 10.1007/978-3-319-60438-1_66 . hal-01511909

\author{
HAL Id: hal-01511909 \\ https://hal.inria.fr/hal-01511909
}

Submitted on 21 Apr 2017

HAL is a multi-disciplinary open access archive for the deposit and dissemination of scientific research documents, whether they are published or not. The documents may come from teaching and research institutions in France or abroad, or from public or private research centers.
L'archive ouverte pluridisciplinaire HAL, est destinée au dépôt et à la diffusion de documents scientifiques de niveau recherche, publiés ou non, émanant des établissements d'enseignement et de recherche français ou étrangers, des laboratoires publics ou privés. 


\title{
Using Formal Concept Analysis for Checking the Structure of an Ontology in LOD: the Example of DBpedia
}

\author{
Pierre Monnin ${ }^{1}$, Mario Lezoche ${ }^{2}$, Amedeo Napoli $^{1}$, and Adrien Coulet ${ }^{1}$ \\ 1 LORIA (CNRS, Inria NGE, Université de Lorraine), Vandœuvre-lès-Nancy, France, \\ 2 CRAN (CNRS, Université de Lorraine), Vandœuvre-lès-Nancy, France
}

\begin{abstract}
Linked Open Data (LOD) constitute a large and growing collection of inter-domain data sets. LOD are represented as RDF graphs that allow interlinking with ontologies, facilitating data integration, knowledge engineering and in a certain sense knowledge discovery. However, ontologies associated with LOD are of different quality and not necessarily adapted to all data sets under study. In this paper, we propose an original approach, based on Formal Concept Analysis (FCA), which builds an optimal lattice-based structure for classifying RDF resources w.r.t. their predicates. We introduce the notion of lattice annotation, which enables comparing our classification with an ontology schema, to confirm subsumption axioms or suggest new ones. We conducted experiments on the DBpedia data set and its domain ontologies, DBpedia Ontology and YAGO. Results show that our approach is well-founded and illustrates the ability of FCA to guide a possible structuring of LOD.
\end{abstract}

Keywords: Linked Open Data, Formal Concept Analysis, classification, ontology engineering

\section{Introduction}

Linked Open Data (LOD) are resulting from a community effort for building a web of data, where data resources may be freely accessed and interpreted by human or software agents for various problem-solving activities. LOD are composed of a large and growing collection of data sets represented within Semantic Web standards that include the use of RDF (Resource Description Framework) and URIs (Uniform Resource Identifiers). In LOD, resources are identified by a URI and can represent entities of the real world (e.g., persons, organizations, places). RDF statements link resources to other resources or to literals (e.g. strings, numbers) using properties, also called predicates. Predicates can be used to link resources from the same data set, from different data sets, or from a data set and an ontology [3. Here, an ontology is a formal representation of a particular domain consisting of classes and relationships between classes. Indeed, resources of a data set may be typed by ontology classes, resulting in class instantiation.

In this paper, we consider DBpedia, an RDF data set of the LOD, built from Wikipedia [12. Wikipedia consists of a very large number of articles, mostly 
composed of plain text, but that may also include structured data as ones in infoboxes. For example, in the Wikipedia article about the French philosopher Voltair ${ }^{3}$, the infobox includes his birth date, his nationality and his occupation. The DBpedia project aims at extracting this structured content and making it available respecting Semantic Web standards. In DBpedia, each Wikipedia article is represented as a DBpedia page. Each page can instantiate multiple classes from several ontologies of the DBpedia data set.

Because of their rapid growth and popularity, LOD data sets and their associated ontologies are of various quality and completeness [16. Consequently, several research papers aim at correcting and completing LOD data sets and ontologies [14]. Some of the proposed approaches extract association rules that express relations between variables, e.g., implications (see 13 for more details on association rule mining with FCA). For example, D'Amato et al. mine both data and an associated ontology to learn assertional axioms (e.g., class instantiations) using association rule mining [5]. AMIE and AMIE+ use Inductive Logic Programming to mine association rules over large knowledge bases composing the LOD [6]7. Alternatively, Alam et al. use FCA to mine DBpedia and obtain implications, subsequently transformed into definitions of ontology classes [1].

In this paper, we compare the structure of an ontology with a hierarchical structure built from RDF resources and the predicates they are subject of. Thanks to this comparison, we are able to check if the structure of the ontology is in agreement with regularities in RDF data. For this purpose, we use Formal Concept Analysis (FCA), which is a mathematical framework well adapted to data analysis and knowledge engineering purposes 2[4. First, we propose applying standard FCA to classify RDF resources within a concept lattice w.r.t. their predicates. Second, we propose to extend FCA with the definition of the annotation of a concept, which associates concepts of the lattice with classes of an ontology. Finally, because they have similar properties to intents, annotations yield rules that we use for checking axioms in ontologies. In the following, we assume that the reader is familiar with the basics of FCA that can be found in [9].

The paper is organized as follows. Section 2 introduces LOD, ontologies and DBpedia. Section 3 presents the method we propose for checking the structure of an ontology. Section 4 details an experimentation with DBpedia data. Finally, we conclude in Section 5 and 6 with a discussion on how FCA could contribute to the structuring of LOD.

\section{Preliminaries}

\subsection{Linked Open Data (LOD) and Ontologies}

Semantic web standards facilitate the publication of LOD online and their connection to existing data sets 3. Indeed, LOD are represented in the form of

\footnotetext{
${ }^{3}$ Wikipedia article about Voltaire: https://en.wikipedia.org/wiki/Voltaire
} 
graphs encoded using RDF. Atomic elements of an RDF graph are triples denoted by:

$$
\langle\text { subject, predicate, object }\rangle \in(U \cup B) \times(U \cup B) \times(U \cup B \cup L)
$$

where $U$ is the set of URIs, $L$ is the set of literals and $B$ represent blank nodes. We note that $B$ is absent in DBpedia.

Ontologies consist of classes and relationships between these classes [10. In this paper, we are interested in the subsumption relation. It is a transitive relation denoted by $\sqsubseteq$, where $\mathrm{c} \sqsubseteq \mathrm{d}$ means that $\mathrm{c}$ is a subclass of $\mathrm{d}$. LOD resources can be linked to several ontologies, i.e., a subject can instantiate one or several classes from one or several ontologies. The semantics of the subsumption relation as well as the semantics of the instantiation of a class by a resource depend on the representation formalism of the ontology. Indeed, taxonomies expressed with SKOS (Simple Knowledge Organization System) use the skos:broader predicate to express subsumption relations and dcterms : subject to express instantiations. Alternatively, ontologies using RDFS (RDF Schema) express subsumption relations using the $\mathrm{rdf} \mathrm{s}$ : subClassOf predicate and instantiations using the rdf: type predicate. For example, the DBpedia page Voltaire is typed with the class Writer from the DBpedia Ontology with the following triple:

$$
\frac{\langle\text { http://dbpedia.org/page/Voltaire, }}{\text { http:type, }}
$$

In this paper, we consider that we have at our disposal an abstract ontology $\mathcal{O}$ using abstract:subClassOf to express subsumption relations and abstract: type to express instantiations. We denote $\mathcal{C}_{\mathcal{O}}$ the set of classes of $\mathcal{O}$. We define the type of a resource e as the set of classes of $\mathcal{O}$ that e instantiates. Formally,

$$
\operatorname{type}(e)=\left\{c \in \mathcal{C}_{\mathcal{O}} \mid\langle e, \text { abstract }: \text { type, } c\rangle\right\}
$$

Accordingly to the definition of the subsumption, an instance of a class $c$ is also an instance of all the classes subsuming $\mathrm{c}$ in $\mathcal{C}_{\mathcal{O}}$. Thus, we define the extended type of e as the whole set of superclasses of the classes of type(e). Formally,

$$
\operatorname{extdtype}(\mathrm{e})=\operatorname{type}(\mathrm{e}) \cup\left\{\mathrm{d} \in \mathcal{C}_{\mathcal{O}} \mid \exists \mathrm{c} \in \operatorname{type}(\mathrm{e}), \mathrm{c} \sqsubseteq \mathrm{d}\right\}
$$

\subsection{DBpedia}

Our study focuses on DBpedia as it is a large LOD data set and as it is associated with ontologies. Particularly, we are interested in DBpedia pages and their classifications w.r.t. available ontologies in DBpedia.

Each DBpedia page is a RDF resource representing an article from Wikipedia. DBpedia pages instantiate classes from multiple ontologies encoded with various languages such as SKOS or RDFS. We consider, in our experiments, the DBpedia Ontology [12] and YAGO [15], which are both encoded in RDFS. The DBpedia Ontology has been manually created based on the most common structured contents available in Wikipedia. YAGO facts have been automatically extracted from Wikipedia and WordNet and their accuracy has been manually evaluated. 


\section{Bridging a Concept Lattice and an Ontology}

Although our experiments were performed on DBpedia, we explain our approach considering the example of an abstract RDF data set. It is composed of the RDF triples from Table 1 containing 4 different resources that are subjects of various triples. We also consider an abstract ontology $\mathcal{O}$ (Figure 22) composed of (i) classes that are instantiated by the subjects of the triples and (ii) subsumption relationships between these classes.

\subsection{Construction of the Concept Lattice}

We build a formal context $(G, M, I)$, where formal objects $G$ are subjects of triples in our data set and attributes $M$ are predicates of the same triples. The incidence relation $I$ indicates that there exists at least one triple where a subject and a predicate appear simultaneously. Here, only two elements of the triples are considered: the subject and the predicate, while the object is not taken into account. Then, we apply standard FCA on this context to build a concept lattice. For example, Table 1 shows RDF triples and the associated formal context. The resulting formal lattice is displayed in Figure 1 .

Table 1. Example of RDF triples and the associated formal context. Triples are represented using the Turtle syntax.

\begin{tabular}{|c|c|c|}
\hline$e_{1}$ & rdf :type & $\mathrm{k}_{1}, \mathrm{k}_{2}$ \\
\hline$e_{1}$ & pred $_{1}$ & $\mathrm{o}_{1}$ \\
\hline$e_{1}$ & pred $_{2}$ & $\mathrm{O}_{2}$ \\
\hline$e_{2}$ & rdf : type & $\mathrm{k}_{1}, \mathrm{k}_{2}, \mathrm{k}_{4}, \mathrm{k}_{5}$ \\
\hline$e_{2}$ & pred $_{1}$ & $\mathrm{O}_{3}$. \\
\hline$e_{2}$ & pred $_{2}$ & $\mathrm{O}_{4}$. \\
\hline$e_{2}$ & pred $_{3}$ & $\mathrm{O}_{5}$ \\
\hline$e_{3}$ & rdf : type & $\mathrm{k}_{1}, \mathrm{k}_{2}$ \\
\hline$e_{3}$ & pred $_{1}$ & $\mathrm{O}_{6}$ \\
\hline$e_{4}$ & rdf : type & $\mathrm{k}_{1}, \mathrm{k}_{2}, \mathrm{k}_{5}$ \\
\hline$e_{4}$ & pred $_{2}$ & $\mathrm{O}_{7}$ \\
\hline$e_{4}$ & pred $_{3}$ & $\mathrm{O}_{8}$ \\
\hline
\end{tabular}

\begin{tabular}{|c|c|c|c|c|}
\hline & rdf : type & pred $_{1}$ & pred $_{2}$ & pred $_{3}$ \\
\hline $\mathrm{e}_{1}$ & $\times$ & $\times$ & $\times$ & \\
\hline $\mathrm{e}_{2}$ & $\times$ & $\times$ & $\times$ & $\times$ \\
\hline $\mathrm{e}_{3}$ & $\times$ & $\times$ & & \\
\hline $\mathrm{e}_{4}$ & $\times$ & & $\times$ & $\times$ \\
\hline
\end{tabular}

\subsection{Annotation of Formal Concepts}

In the lattice in Figure 1 each extent contains subjects and each intent contains predicates. To compare the lattice with the ontology $\mathcal{O}$, we need to "link" formal concepts with ontology classes. To do so, given $A \subseteq G$ and $K \subseteq \mathcal{C}_{\mathcal{O}}$, we define two new dual derivation operators denoted by $(\cdot)^{\diamond}: 2^{G} \rightarrow 2^{\mathcal{C}_{\mathcal{O}}}$ and $(\cdot)^{\diamond}: 2^{\mathcal{C}_{\mathcal{O}}} \rightarrow 2^{G}$ such as:

$$
A^{\diamond}=\bigcap_{\mathrm{e} \in A} \operatorname{extdtype}(\mathrm{e}) \quad \text { and } \quad K^{\diamond}=\{\mathrm{e} \in G \mid K \subseteq \operatorname{extdtype}(\mathrm{e})\}
$$




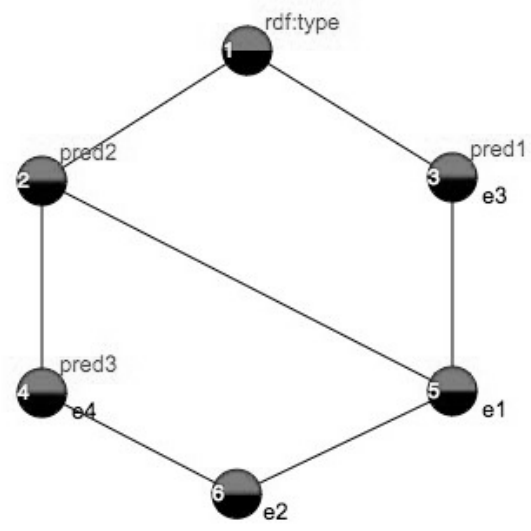

Fig. 1. Line diagram representing the concept lattice built from the formal context in Table 1. The lattice is displayed using the reduced notation. Following this notation, formal objects, depicted in black, are associated with one concept and, implicitly, with all its superconcepts. Attributes, in grey, are associated with one concept and, implicitly, with all its subconcepts. Formal concepts are arbitrarily numbered from 1 to 6 .

$A^{\diamond}$ contains the ontology classes shared by the extended types of all the subjects in $A . K^{\diamond}$ represents the set of subjects having all the ontology classes of $K$ in their extended type. We prove in Appendix that $(\cdot)^{\triangleright \diamond}: 2^{\mathcal{C}_{\mathcal{O}}} \rightarrow 2^{\mathcal{C}_{\mathcal{O}}}$ and $(\cdot)^{\diamond}: 2^{G} \rightarrow 2^{G}$ are closure operators.

Given a formal concept $(A, B)$, we call $A^{\diamond}$ the annotation of the concept and we define the triple $\left(A, B, A^{\diamond}\right)$ as an annotated concept. For example, consider the concept $4\left(\left\{\mathrm{e}_{2}, \mathrm{e}_{4}\right\},\left\{\mathrm{rdf}: \mathrm{type}_{\mathrm{p}} \mathrm{pred}_{2}, \mathrm{pred}_{3}\right\}\right)$ in Figure 1. Based on the ontology in Figure 2, we have $\left\{\mathrm{e}_{2}, \mathrm{e}_{4}\right\}^{\diamond}=\left\{\mathrm{k}_{1}, \mathrm{k}_{2}, \mathrm{k}_{3}, \mathrm{k}_{5}\right\}$. So, the annotated concept is $\left(\left\{\mathrm{e}_{2}, \mathrm{e}_{4}\right\},\left\{\mathrm{rdf}:\right.\right.$ type, $\left.\left.\operatorname{pred}_{2}, \operatorname{pred}_{3}\right\},\left\{\mathrm{k}_{1}, \mathrm{k}_{2}, \mathrm{k}_{3}, \mathrm{k}_{5}\right\}\right)$. Accordingly, we define the annotated lattice as the lattice where each concept is replaced by its corresponding annotated concept.

Given two concepts such as $\left(A_{1}, B_{1}\right) \leqslant\left(A_{2}, B_{2}\right)$. As $A_{1} \subseteq A_{2}$, we have $A_{2}^{\diamond} \subseteq A_{1}^{\diamond}$ (see Equation (1) in Appendix). Therefore, we also extend the reduced notation to represent an annotated lattice: extents and intents are depicted as usual and annotations are depicted showing only the new classes that are not already in the annotations of the superconcepts. For example, on the annotated lattice in Figure 3, the annotation of the concept 4 shows only $k_{5}$ because $k_{1}, k_{2}$ and $\mathrm{k}_{3}$ are already in the annotation of its superconcepts.

\subsection{Comparing Rules Extracted in the Lattice and Axioms in the Ontology}

In the annotated lattice in Figure 3, extents contain subjects, intents contain predicates and annotations contain classes of $\mathcal{O}$. To check the structure of the 


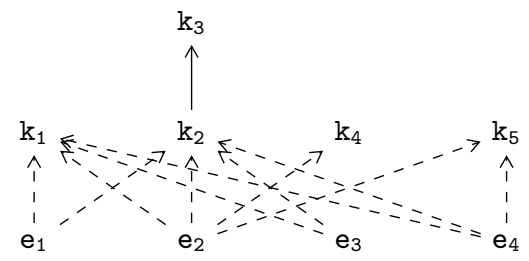

Fig. 2. Example of ontology classes being instantiated by subjects. Dotted arrows represent instantiations whereas solid arrows represent subsumption relations. For example, $\mathrm{e}_{1}$ instantiates $\mathrm{k}_{1}$ and $\mathrm{k}_{2}$, and $\mathrm{k}_{2}$ is subsumed by $\mathrm{k}_{3}$.

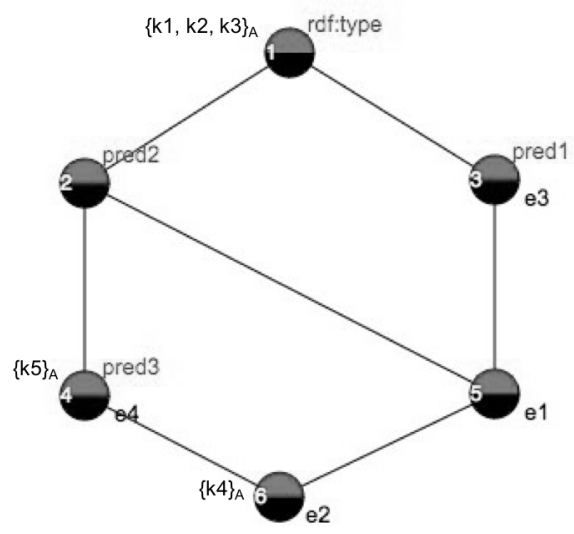

Fig. 3. Line diagram representing the annotated lattice based on the concept lattice in Figure 1 and the ontology in Figure 2. It is displayed using our extension of the reduced notation. Formal objects are depicted in black, attributes in grey and annotations are depicted by $\{\cdot\}_{A}$. 
ontology, in other words, to check the subsumption axioms existing between classes of $\mathcal{O}$, we have to extract rules from the lattice that can be considered similar to subsumption axioms. To do so, we consider each pair composed of an annotated concept $\left(A, B, A^{\diamond}\right)$ and one of its covering concept $\left(E, F, E^{\diamond}\right)$, i.e. one of its direct superconcepts, such as the pair composed of concepts 6 and 4 in Figure 3. Similarly to Figure 3, we denote $A_{A}^{\diamond}=\left\{\mathrm{x}_{1}, \mathrm{x}_{2}, \ldots, \mathrm{x}_{\mathrm{p}}\right\}$ and $E_{A}^{\diamond}=\left\{\mathrm{y}_{1}, \mathrm{y}_{2}, \ldots, \mathrm{y}_{\mathrm{q}}\right\}$ the reduced notation of the annotations $A^{\diamond}$ and $E^{\diamond}$.

Then, we consider each pair of ontology classes $\left(\mathrm{x}_{\mathrm{i}}, \mathrm{y}_{\mathrm{j}}\right)$ from $A_{A}^{\diamond} \times E_{A}^{\diamond}$. Considering concepts 6 and 4 , the only pair that can be considered is $\left(\mathrm{k}_{4}, \mathrm{k}_{5}\right)$. Because $A \subseteq E$ and $E^{\diamond} \subseteq A^{\diamond}$, we can say that all subjects typed by $\mathrm{x}_{\mathrm{i}}$ are also typed by $\mathrm{y}_{\mathrm{j}}$. Moreover, $\mathrm{y}_{\mathrm{j}}$ can type subjects that are not typed by $\mathrm{x}_{\mathrm{i}}$. Hence, $\mathrm{x}_{\mathrm{i}}$ can be viewed as "more specialized" than $\mathrm{y}_{j}$. This can be interpreted as follows: the annotated lattice "suggests" $x_{i}$ as a subclass of $y_{j}$. Because we use the cover relation, we consider this suggestion as a direct subclass suggestion. Here, $k_{4}$ is suggested as a direct subclass of $k_{5}$.

This rule extracted from the lattice is then compared with the subsumption axioms defined in $\mathcal{O}$ :

- If $x_{i}$ is already explicitly declared as a subclass of $y_{j}$, we call this rule a confirmed axiom.

- If $\mathrm{x}_{\mathrm{i}}$ is an indirect subclass of $\mathrm{y}_{\mathrm{j}}$, i.e. $\mathrm{x}_{\mathrm{i}}$ is not explicitly stated as a subclass of $\mathrm{y}_{\mathrm{j}}$ but it can be inferred from $\mathcal{O}$ thanks to the transitivity of the subsumption relation, we call the rule an inferable axiom.

- Otherwise, the rule does not exist in the ontology and we consider it as a potential new axiom, proposed to improve the ontology.

In our example, according to the ontology in Figure 2 , there is no link existing between $k_{4}$ and $k_{5}$. Thus, $k_{4} \sqsubseteq k_{5}$ is a new axiom proposal.

If we consider the pair composed of concepts 6 and 5 in Figure 3 , we notice that the reduced notation of the annotation of concept 5 is empty. Thus, no rule can be extracted from this pair of concepts. Similarly, no rule involving $\mathrm{k}_{1}, \mathrm{k}_{2}$ and $k_{3}$ can be extracted with the current approach because the reduced notation of the annotations of concepts 2 and 3 are empty.

\section{Experimentation}

We experimented this approach, classifying 904 pages selected from the DBpedia 2014 data set that describe persons who died between 01/01/2000 and 07/01/2000 (included). The resulting lattice consisted of 15,234 concepts. Axioms from the DBpedia Ontology (11) and YAGO (199) were retrieved among the rules and, therefore, were considered as confirmed axioms. For example, the DBpedia Ontology class Boxer was found as a subclass of Athlete. Inferable axioms $(2,250)$ as well as new axioms $(1,372)$ were only found for YAGO. This may be due to the small size of the DBpedia Ontology which only contains 683 classes. Therefore, pages have more similar extended types than with a more 
fine-grained ontology such as YAGO. Consequently, annotations of formal concepts are also similar, leading to empty reduced notation of annotations from which no rule is extracted (see the example with concepts 5 and 6 in Figure 3). For example, the lattice we obtained from our subset of DBpedia pages suggested to add BostonUniversityAlumni as a subclass of Scholar110557854. Nevertheless, this subsumption could be inferred from the ontology. Another example was the suggestion of FilipinoChildActors as a subclass of FilipinoActors. It was neither explicitly stated in the ontology nor inferable and seemed to make sense in a general case.

\section{Discussion}

Regarding the results of the experiments, when classifying RDF subjects w.r.t. their related predicates, we are able to suggest direct subclass relations between ontology classes typing these subjects. Some of the suggestions already exist as direct subclass relations in the considered ontology. Thus, predicates allow to classify subjects in a lattice whose structure is similar to some parts of the existing ontology. Consequently, it seems that some predicates applied to subjects are specific of the classes typing these subjects. Therefore, predicates could be considered as "indicators" of the classification of subjects by ontology classes. Some of the suggested axioms indirectly exist in the ontology, meaning that the structure of the lattice is not as fine-grained as the ontology. This may be due to the fact that predicates applied to subjects are not the only indicators of their classification by classes of an ontology. In the annotated lattice in Figure 3 , we also notice that the reduced notation of the annotation is empty for concepts 2,3 and 5 because these concepts are not closed w.r.t. the $(\cdot)^{\infty}$ operator. Therefore, we do not extract rules from them, in addition the classes $k_{1}, k_{2}$ and $k_{3}$ never appear in rules extracted from the lattice.

As future work, we will consider additional features in classification to provide more granularity. For example, the formal context could be built using predicates, (predicate, extdtype(object)) and (predicate, object) pairs as attributes. Pattern Structures [8] could be used on extended types as there is a partial order on classes of an ontology. Because $(\cdot)^{\diamond}$ is a closure operator, we could also use the triadic approach to FCA [11] to model the three sets that describe each of our concepts (extents, intents and annotations). Another perspective of the current work is to reduce the lattice by keeping only the closed concepts relatively to the $(\cdot)^{\infty}$ operator. This will increase the number of suggestions. For example, in Figure 3 removing concepts 2, 3 and 5 leads to suggest $k_{5}$ and $k_{4}$ as subclasses of $k_{1}, k_{2}$ and $k_{3}$. Finally, in the case of subjects typed by two (or more) ontologies, two annotations can be computed for each concept of the lattice, providing potential relationships between independent ontologies. In this case, the lattice can be seen as a pivot structure for ontology matching experiments. 


\section{Conclusion}

In this paper, we apply FCA to classify RDF subjects w.r.t. their related predicates in a concept lattice. A method is proposed to annotate this lattice with ontology classes used to type these subjects. Based on the structure of the resulting lattice, subsumption axioms between the ontology classes are suggested and then compared with existing axioms in the ontology. Running this method on a subset of pages from DBpedia and considering two associated reference ontologies (DBpedia Ontology and YAGO), we are able to suggest axioms that already exist. This means that the structure of the annotated lattice is similar to some parts of the existing ontologies. Consequently, predicates applied to pages can be considered as indicators of classes instantiated by these pages. New axioms are also proposed. One of the next challenges resides in performing a qualitative study of these new axioms.

\section{Appendix: Proving that $(\cdot)^{\diamond \diamond}$ is a Closure Operator}

An operator on a partial ordered set is a closure operator if it is monotonous, extensive and idempotent. Firstly, let us prove that:

$$
X_{1} \subseteq X_{2} \Rightarrow X_{2}^{\diamond} \subseteq X_{1}^{\diamond}
$$

To do so, let's consider $E_{1} \subseteq G$ and $E_{2} \subseteq G$ such as $E_{1} \subseteq E_{2}$ and, dually, $K_{1} \subseteq \mathcal{C}_{\mathcal{O}}$ and $K_{2} \subseteq \mathcal{C}_{\mathcal{O}}$ such as $K_{1} \subseteq K_{2}$. We have:

$$
\begin{aligned}
E_{2}^{\diamond} & =\bigcap_{\mathrm{e} \in E_{2}} \operatorname{extdtype}(\mathrm{e})=\left(\cap_{\mathrm{e} \in E_{1}} \operatorname{extdtype}(\mathrm{e})\right) \bigcap\left(\cap_{\left.\mathrm{e} \in E_{2} \backslash E_{1} \operatorname{extdtype}(\mathrm{e})\right)}\right. \\
& =E_{1}^{\diamond} \cap\left(\cap_{\mathrm{e} \in E_{2} \backslash E_{1}} \operatorname{extdtype}(\mathrm{e})\right) \subseteq E_{1}^{\diamond} \\
K_{2}^{\diamond} & =\left\{\mathrm{e} \in G \mid K_{2} \subseteq \operatorname{extdtype}(\mathrm{e})\right\} \\
& =\left\{\mathrm{e} \in G \mid K_{1} \subseteq \operatorname{extdtype}(\mathrm{e}) \wedge K_{2} \backslash K_{1} \subseteq \operatorname{extdtype}(\mathrm{e})\right\} \\
& =\left\{\mathrm{e} \in G \mid K_{1} \subseteq \operatorname{extdtype}(\mathrm{e})\right\} \bigcap\left\{\mathrm{e} \in G \mid K_{2} \backslash K_{1} \subseteq \operatorname{extdtype}(\mathrm{e})\right\} \\
& =K_{1}^{\diamond} \bigcap\left\{\mathrm{e} \in G \mid K_{2} \backslash K_{1} \subseteq \operatorname{extdtype}(\mathrm{e})\right\} \subseteq K_{1}^{\diamond}
\end{aligned}
$$

Monotonicity. Considering $X_{1} \subseteq X_{2}$. Because of (1), we have $X_{2}^{\diamond} \subseteq X_{1}^{\diamond}$ and then $X_{1}^{\diamond \diamond} \subseteq X_{2}^{\diamond \diamond}$.

Extensivity. Considering $E \subseteq G$, we have $E^{\diamond}=\bigcap_{\mathrm{e} \in E} \operatorname{extdtype}(\mathrm{e})$. So, $E^{\diamond \diamond}=\left\{\mathrm{f} \in G \mid \bigcap_{\mathrm{e} \in E} \operatorname{extdtype}(\mathrm{e}) \subseteq \operatorname{extdtype}(\mathrm{f})\right\}$. Therefore, $E \subseteq E^{\diamond \diamond}$. Dually,

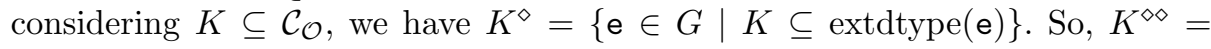

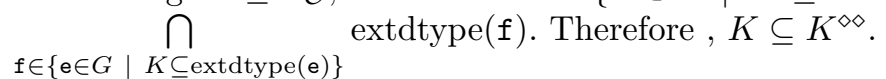


Idempotence. Because of the extensivity, we know that $X \subseteq X^{\diamond}$. Therefore, because of (1), $X^{\diamond \diamond \diamond} \subseteq X^{\diamond}$ and $X^{\diamond \diamond} \subseteq X^{\diamond \diamond \diamond \diamond}$. Because of the extensivity, $X^{\diamond} \subseteq$ $X^{\diamond \diamond \diamond}$. So because of (1), $X^{\infty \diamond \diamond \diamond} \subseteq X^{\diamond \diamond}$. Consequently, $X^{\diamond \diamond}=X^{\diamond \diamond \diamond}$.

\section{References}

1. Alam, M., Buzmakov, A., Codocedo, V., Napoli, A.: Mining definitions from RDF Annotations Using Formal Concept Analysis. In: Proceedings of IJCAI 2015. pp. 823-829 (2015)

2. Bendaoud, R., Napoli, A., Toussaint, Y.: Formal Concept Analysis: A Unified Framework for Building and Refining Ontologies. In: EKAW 2008. Proceedings. pp. $156-171$ (2008)

3. Bizer, C., Heath, T., Berners-Lee, T.: Linked Data - The Story So Far. Int. J. Semantic Web Inf. Syst. 5(3), 1-22 (2009)

4. Cimiano, P., Hotho, A., Staab, S.: Learning Concept Hierarchies from Text Corpora using Formal Concept Analysis. J. Artif. Intell. Res. (JAIR) 24, 305-339 (2005)

5. d'Amato, C., Staab, S., Tettamanzi, A.G., Minh, T.D., Gandon, F.: Ontology enrichment by discovering multi-relational association rules from ontological knowledge bases. In: Proceedings of ACM SAC'16. pp. 333-338 (2016)

6. Galárraga, L., Teflioudi, C., Hose, K., Suchanek, F.M.: Fast rule mining in ontological knowledge bases with AMIE+. VLDB J. 24(6), 707-730 (2015)

7. Galárraga, L.A., Teflioudi, C., Hose, K., Suchanek, F.: AMIE: association rule mining under incomplete evidence in ontological knowledge bases. In: WWW'13. pp. $413-422(2013)$

8. Ganter, B., Kuznetsov, S.O.: Pattern structures and their projections. In: International Conference on Conceptual Structures. pp. 129-142. Springer (2001)

9. Ganter, B., Wille, R.: Formal Concept Analysis: Mathematical Foundations. Springer (1999)

10. Gruber, T.R., et al.: A translation approach to portable ontology specifications. Knowledge acquisition 5(2), 199-220 (1993)

11. Lehmann, F., Wille, R.: A triadic approach to formal concept analysis. Conceptual structures: applications, implementation and theory pp. 32-43 (1995)

12. Lehmann, J., et al.: DBpedia - A large-scale, multilingual knowledge base extracted from wikipedia. Semantic Web 6(2), 167-195 (2015)

13. Pasquier, N., Bastide, Y., Taouil, R., Lakhal, L.: Efficient mining of association rules using closed itemset lattices. Information systems 24(1), 25-46 (1999)

14. Paulheim, H.: Automatic Knowledge Graph Refinement: A Survey of Approaches and Evaluation Methods. Semantic Web Journal (To appear)

15. Suchanek, F.M., Kasneci, G., Weikum, G.: YAGO: A Core of Semantic Knowledge Unifying WordNet and Wikipedia. In: Proceedings of WWW'07. pp. 697-706. ACM (2007)

16. Zaveri, A., Rula, A., Maurino, A., Pietrobon, R., Lehmann, J., Auer, S.: Quality assessment for Linked Data: A Survey. Semantic Web 7(1), 63-93 (2016) 